\title{
A multi-center phase II study of high dose interleukin-2 sequenced with vemurafenib in patients with BRAF-V600 mutation positive metastatic melanoma
}

Joseph I. Clark ${ }^{1 *}$ D, Jatinder Singh ${ }^{2}$, Marc S. Ernstoff ${ }^{3}$, Christopher D. Lao ${ }^{4}$, Lawrence E. Flaherty ${ }^{5}$,

Theodore F. Logan ${ }^{6}$, Brendan Curti ${ }^{7}$, Sanjiv S. Agarwala ${ }^{8}$, Bret Taback ${ }^{9}$, Lee Cranmer ${ }^{10}$, Jose Lutzky ${ }^{11}$,

Theresa L. Luna ${ }^{12}$, Sandra Aung ${ }^{12,14}$ and David H. Lawson ${ }^{13}$

\begin{abstract}
Background: Preclinical studies suggest that BRAF inhibitors enhance anti-tumor immunity and antigen presentation. Combination BRAF inhibition with immunotherapy is an appealing therapeutic approach. We sequenced vemurafenib with HD IL-2 in patients with BRAF-mutated metastatic melanoma to improve long term outcomes.

Methods: Eligible patients were HD IL-2 eligible with metastatic BRAF V600 mutated melanoma. Cohort 1 was treatment naïve and received vemurafenib $960 \mathrm{mg}$ BID for 6 weeks before HD IL-2. Cohort 2 received vemurafenib for 7-18 weeks before enrollment. Both cohorts received HD IL-2 at 600,000 IU/kg every $8 \mathrm{~h}$ days $1-5$ and days 1519. The primary objective was to assess complete responses (CR) at 10 weeks \pm 3 (assessment 1 ) and 26 weeks \pm 3 (assessment 2) from the start of HD IL-2.
\end{abstract}

Results: Fifty-three patients were enrolled, (cohort 1, $n=38$; cohort 2, $n=15$ ). Of these, 39 underwent assessment 1 and 15 assessment 2. The CR rate at assessment 1 was 10\% (95\% Cl 3-24) for both cohorts combined, and 27\% (95\% Cl 8-55) at assessment 2. Three-year survival was 30 and 27\% for cohort 1 and cohort 2, respectively. No unexpected toxicities occurred. A shift in the melanoma treatment landscape during this trial adversely affected accrual, leading to early trial closure.

Conclusions: Vemurafenib in sequence with HD IL-2 did not change the known toxicity profile for either agent. Lower than expected response rates to vemurafenib were observed. Overall response rates and durability of responses appear similar to that observed with HD IL-2 alone.

Trial registration: NCTN, NCT01683188. Registered 11 September 2012, http://www.clinicaltrials.gov/NCT01683188 Keywords: High-dose interleukin-2, Vemurafenib, BRAF-mutated metastatic melanoma, Multicenter, Phase II

\footnotetext{
* Correspondence: jclark@lumc.edu

${ }^{1}$ Cardinal Bernardin Cancer Center, Loyola University Medical Center, 2160 S.

First Avenue, Maywood, IL 60153, USA

Full list of author information is available at the end of the article
}

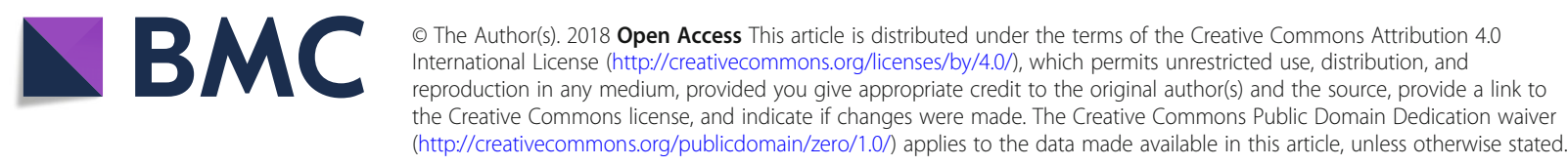




\section{Background}

Treatment of metastatic malignant melanoma has dramatically advanced with the development of targeted agents and immunotherapy in the recent past. Targeting the MAPK pathway with BRAF inhibitors, MEK inhibitors, or the combination, leads to high response rates and progression free survivals of roughly 7 months for single agents [1-3], to about 11 months for combination regimens [4, 5]. Approved immunotherapies now include high dose interleukin-2 (HD IL-2) and the immune checkpoint inhibitors, (ipilimumab, nivolumab, pembrolizumab). Durable responses are consistently observed in a small percentage of patients with metastatic melanoma treated with HD IL-2 [6-8]. Higher response rates are observed with immune checkpoint inhibitors [9-13], especially with combination anti-CTLA and anti-PD1 agents [14-16], but longer follow up is required to determine the durability of these responses and more recent studies report acquired resistance to these agents [17].

Preclinical studies suggest that oncogenic BRAF (BRAF V600E) may contribute to immune escape in melanoma [18], and that blocking its activity via MAPK inhibition leads to increased expression of melanocytic differentiation antigens (MDAs) $[19,20]$ with significantly enhanced recognition by antigen-specific $\mathrm{T}$ lymphocytes $[20,21]$, enhanced antigen presentation $[22,23]$, without diminishing T-cell function [24], and change in the tumor-produced immune environment [25]. The combination of BRAF inhibition with systemic immunotherapy is therefore an appealing therapeutic approach in the treatment of patients with advanced melanoma. That is, the effects of BRAF inhibition, including disease reduction and control for several months may enhance the likelihood of complete response to HD IL-2 therapy with subsequent anticipated improved durability of these responses.

In this open-label phase 2 trial we sequenced the BRAF inhibitor vemurafenib with HD IL-2 in the treatment of patients with stage IV, metastatic BRAF-mutated malignant melanoma and assessed the toxicity and efficacy specifically with regard to the complete response rate from this combination.

\section{Methods}

\section{Patients}

Eligible patients were age $\geq 18$ years with histologically confirmed, not surgically resectable due to extent of disease, measurable stage IV BRAF V600E- or V600 K-mutated malignant melanoma. Patients had to meet requirements for HD IL-2 therapy as previously described [6] and vemurafenib therapy per institutional guidelines. Treatment naïve patients were enrolled in Cohort 1; patients who had been receiving active treatment with vemurafenib for 7 to 18 weeks were enrolled in Cohort 2. Patients were excluded if they had received prior treatment with HD
IL-2, ipilimumab or other highly selective BRAF, MEK, NRAS, or cMET inhibitors. Prior treatment with an anti-PD-1 or anti-PDL-1 antibody was allowed. Exclusion also included a prolonged QTc interval of $>500 \mathrm{~ms}$; known or suspected infection with HIV, hepatitis C, hepatitis B or other infectious hepatitis; pregnant or nursing women; untreated brain metastases; prior investigational drug within 30 days. Human investigations were performed after approval by an institutional review board or ethics committee at each participating institution and in accordance with an assurance filed with and approved by the U.S. Department of Health and Human Services. Written informed consent was obtained on all patients.

\section{Study design and treatment}

The study was approved by the institutional review board or ethics committee at each participating institution. Eligible patients who were treatment naïve were enrolled in Cohort 1 and received vemurafenib $960 \mathrm{mg}$ by mouth twice daily for 6 weeks prior to receiving inpatient HD IL-2. Cohort 2 patients included eligible patients who had been receiving vemurafenib therapy for 7 to 18 weeks with stable or responding disease prior to enrollment. Baseline imaging studies were performed prior to starting vemurafenib in Cohort 1 and prior to starting HD IL-2 in Cohort 2. Both cohorts received HD IL-2 at $600,000 \mathrm{IU} / \mathrm{kg}$ intravenously over $15 \mathrm{~min}$ every $8 \mathrm{~h}$ for a maximum of 14 doses days $1-5$, (cycle 1 ), and days 15-19, (cycle 2). Each course consisted of 2 cycles. Vemurafenib was held during inpatient HD IL-2 therapy, (Fig. 1), and resumed during the 9 days between cycles and at the completion of cycle 2. Dose adjustments of vemurafenib or withholding of HD IL-2 doses were performed by the respective treating physicians based on accepted standards of care for each of these agents. QTc intervals were reviewed daily for changes during each 5 day cycle of HD IL-2 dosing.

\section{Study assessments/statistical analysis}

Baseline imaging for cohort 1 occurred within 4 weeks of starting vemurafenib whereas for cohort 2, baseline imaging occurred after patients had been receiving vemurafenib for $>7$ weeks but $<18$ weeks and either an objective response or stable disease had to have been observed before enrollment and initiation of HD IL-2 therapy. Disease response assessment occurred at week $10 \pm 3$ weeks, (assessment 1), and at week $26 \pm 3$ weeks, (assessment 2), from the start of HD IL-2 dosing. Patients received up to 2 courses of HD IL- 2 if evidence of disease response was observed after the first course. Course 2 began at least 8 weeks after the completion of course 1. RECIST and immune-related response criteria (irRC) were used to assess response [26]. 


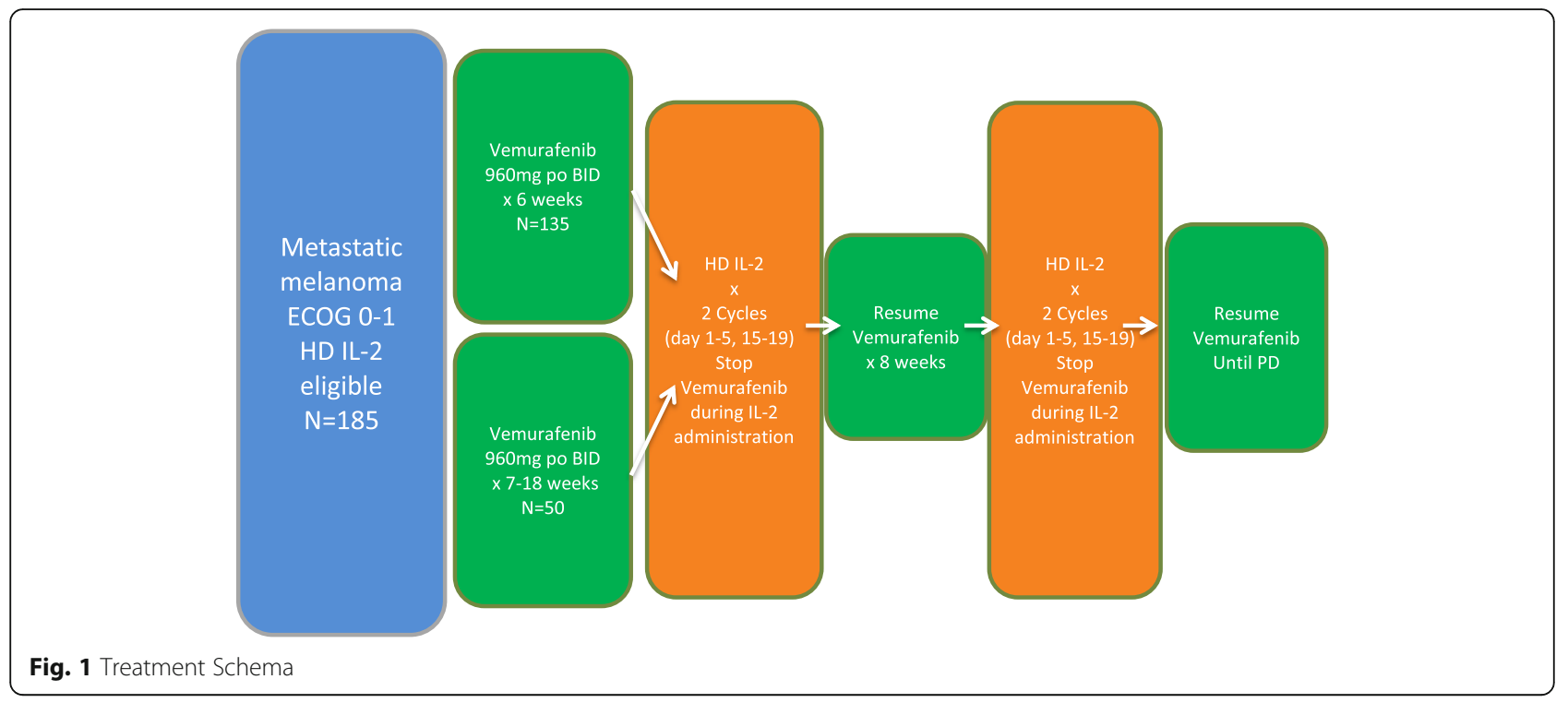

Cohort 1 was used to define the study size and statistical validity with the comparator being historical controls using data from BRAF positive patients from the Melanoma Select study (NCT01288963) [27]. The initial design was to enroll 135 subjects in Cohort 1 . Based on a one-sample binomial test (using normal approximation), a sample size of 135 would have $80 \%$ power to detect a significant difference using a one-sided test with $\alpha=0.05 \%$ if the true complete response (CR) rate for naïve subjects treated with 6 weeks of vemurafenib prior to adding HD IL-2 was twice the $\mathrm{CR}$ rate of the historical control for HD IL-2 (12\% vs $6 \%)$. Up to 50 subjects were allowed to be enrolled in Cohort 2. This cohort was designed to evaluate whether additive or synergistic clinical benefit or toxicity was observed in BRAF-mutated melanoma patients treated with vemurafenib as a single agent for $>7$ to 18 weeks prior to the first course of HD IL-2 therapy in conjunction with continued vemurafenib.

Secondary endpoints included (1) assessment of tumor response in patients with CR or near CR (> 90\%) after discontinuation of vemurafenib, (2) compare progression free survival (PFS) from initiation of vemurafenib between Cohort 1 and Cohort 2 patients, (3) determine the duration of response in patients treated with vemurafenib and HD IL-2, (4) compare overall PFS with the historical data using vemurafenib or HD IL-2 alone, (5) compare safety between Cohort 1 and Cohort 2 patients, (6) compare safety between patients treated with vemurafenib and HD IL-2 versus historical HD IL-2 alone, (7) explore potential biomarkers for their predictive value in combination therapy of vemurafenib and HD IL-2, (8) assess treatment response to retreatment with vemurafenib in "CR" patients progressing on no therapy.

\section{Results}

A total of 53 patients with BRAF V600-mutated metastatic melanoma were enrolled onto this trial. Cohort 1 included 38 patients and cohort 2, 15 patients, none of whom received prior anti-PD1 or anti-PDL1 therapy (Table 1). All evaluable patients received vemurafenib and at least one dose of HD IL-2. Of the 53 patients enrolled, 39 completed assessment 1 and 15 completed assessment 2, respectively. A caveat to this trial required that patients be enrolled into the PROCLAIM registry [27] once they started HD IL-2 therapy, for purposes of capturing survival data. Fourteen patients' data were not captured for assessment 1 for various reasons: seven patients never proceeded on to HD IL-2 treatment, thus they were not entered into the PROCLAIM registry, and as such their response information was not captured. Of these seven, two patients experienced an adverse event on vemurafenib and the decision was made not to proceed with HD IL-2 therapy; one patient withdrew consent before receiving HD IL-2; two patients were unable to continue on protocol treatment due to insurance issues; one patient experienced progression of disease while taking vemurafenib thus did not proceed onto HD IL-2 treatment; one patient chose not to proceed with HD IL-2 treatment. Of the other seven patients who did not undergo assessment 1: in two patients support for the trial terminated prior to assessment 1 and they were treated off study; four patients withdrew consent after starting HD IL-2; one patient experienced early disease progression shortly after starting HD IL-2 therapy.

No difference between RECIST and irRC was observed. The best overall response rate, (ORR) (CR/PR), at assessment 1 was $27 \%$ (95\% CI 12-46) and disease control rate, (CR/PR/SD) was $77 \%$ (95\% CI 58-90) for 
Table 1 Patient Demographics

\begin{tabular}{|c|c|c|}
\hline \multirow[t]{2}{*}{ Characteristic } & Cohort 1 & Cohort 2 \\
\hline & Vemurafenib 6 weeks $(N=38)$ & Vemurafenib 7-18 weeks $(N=15)$ \\
\hline \multicolumn{3}{|l|}{ Age (yr) } \\
\hline Mean (SD) & $48.6(12.79)$ & $48.5(13.90)$ \\
\hline Median & 50.5 & 48.0 \\
\hline Range & $21-73$ & $26-67$ \\
\hline \multicolumn{3}{|l|}{ Gender - no. (\%) } \\
\hline Male & $15(39.5)$ & $6(40.0)$ \\
\hline Female & $23(60.5)$ & $9(60.0)$ \\
\hline \multicolumn{3}{|l|}{ Race - no. (\%) } \\
\hline White & $35(92.1)$ & $15(100.0)$ \\
\hline Decline & $2(5.3)$ & $0(0.0)$ \\
\hline Other & $1(2.6)$ & $0(0.0)$ \\
\hline \multicolumn{3}{|c|}{ Metastasis stage - no. (\%) } \\
\hline M1a & $9(23.7)$ & $2(13.3)$ \\
\hline M1b & $13(34.2)$ & $3(20.0)$ \\
\hline M1c & $16(42.1)$ & $10(66.7)$ \\
\hline \multicolumn{3}{|l|}{ LDH level - no. (\%) } \\
\hline$\leq \cup L N$ & $17(44.7)$ & $5(33.3)$ \\
\hline$>$ ULN & $17(44.7)$ & $8(53.3)$ \\
\hline Not measured & $4(10.5)$ & $2(13.3)$ \\
\hline \multicolumn{3}{|c|}{ Prior therapy - no. (\%) } \\
\hline Surgery & $34(89.5)$ & $13(86.7)$ \\
\hline Radiation & $7(18.4)$ & $8(53.3)$ \\
\hline Chemotherapy & $4(10.5)$ & $1(6.7)$ \\
\hline Anti-PD1/PDL1 & 0 & 0 \\
\hline \multicolumn{3}{|l|}{ Mutation - no. (\%) } \\
\hline BRAF & $38(100.0)$ & $15(100.0)$ \\
\hline CKIT & $1(2.6)$ & $0(0.0)$ \\
\hline Other & $1(2.6)$ & $0(0.0)$ \\
\hline \multicolumn{3}{|l|}{ Performance Status } \\
\hline 0 & $24(63.2)$ & $8(53.3)$ \\
\hline 1 & 14 (36.8) & $7(46.7)$ \\
\hline
\end{tabular}

Race of 'Other' had the description 'Not specified' recorded

Table 2 Best Overall Response

\begin{tabular}{lllll}
\hline Group & Cohort 1 $(N=30)$ & Cohort 2 $(N=15)$ & Assessment 1 $(N=39)$ & Assessment 2 (N=15) \\
\hline Complete Response & $3(10 \%)$ & $1(7 \%)$ & $4(10 \%)$ & $4(27 \%)$ \\
$95 \% \mathrm{Cl}(\%)$ & $(2,27)$ & $(0.2,32)$ & $(3,24)$ & $(8,55)$ \\
Response (CR/PR) & $8(27 \%)$ & $4(27 \%)$ & $11(28 \%)$ & $9(60 \%)$ \\
$95 \% \mathrm{Cl}(\%)$ & $(12,46)$ & $(8,55)$ & $(15,45)$ & $(33,84)$ \\
Disease Control (CR/PR/SD) & $23(77 \%)$ & $11(73 \%)$ & $30(77 \%)$ & $15(100 \%)$ \\
$95 \% \mathrm{Cl}(\%)$ & $(58,90)$ & $(45,92)$ & $(61,89)$ & $(78,100)$ \\
\hline
\end{tabular}




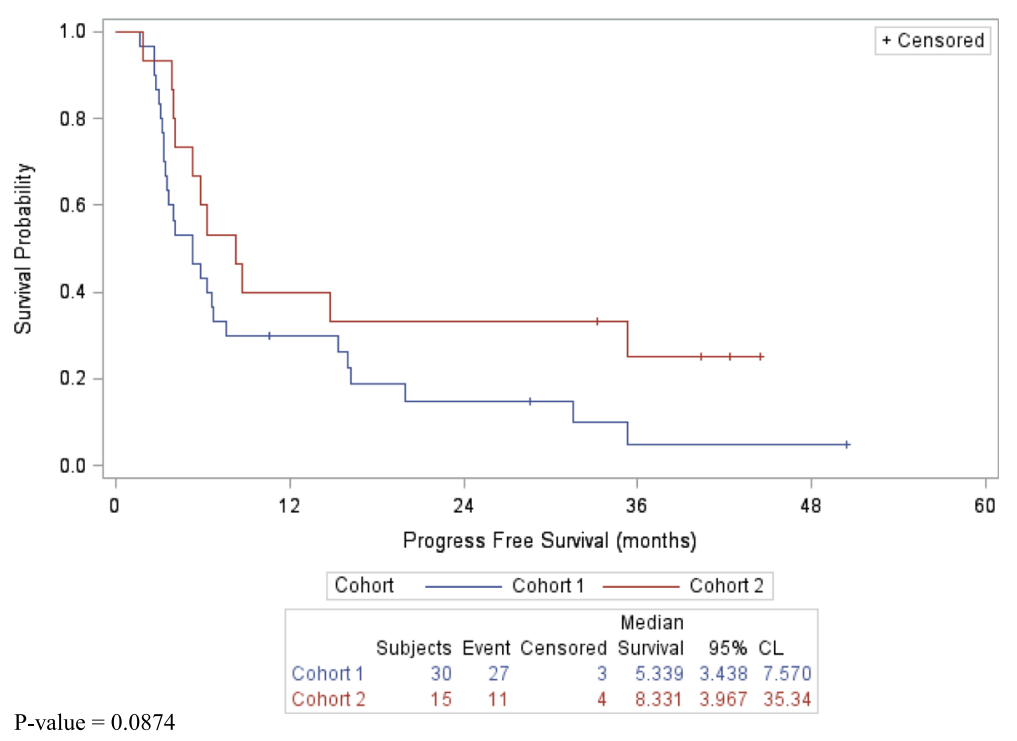

Fig. 2 Progression-Free Survival by Cohort

cohort $1,(n=30)$, and $27 \%(95 \%$ CI $8-55)$ and $73 \%(95 \%$ CI 45-92) for cohort $2(n=15)$, respectively, (Table 2$)$. The CR rate was $10 \%$ (95\% CI 2-17) for cohort 1 and $7 \%$ (95\% CI 0.2-32) for cohort 2. At assessment 2, of the 15 patients who were assessed in both cohorts, the ORR was $60 \%$ (95\% CI 33-84) and the disease control rate was $100 \%$ (95\% CI 78-100). The median PFS was 5.3 months (95\% CI 3.4-7.5) for cohort 1 and 8.3 months (95\% CI 4.0-35.3) for cohort 2, $(p$-value $=0.0874)$, (Fig. 2). The duration of response ranged from 0.27 to $17+$ months for all responders, and for those with stable disease up to $18.1+$ months (Table 3 ). The median OS was 16.1 months (95\% CI, 9.2-34.9) for cohort 1 and 14.9 months (95\% CI 4.8-35.3) for cohort 2, (p-value = 0.9709), (Fig. 3). The 1, 2 and 3-year survivals were similar in both cohorts, (Table 4). No subjects who achieved a CR recurred.

No unexpected toxicities were observed with either vemurafenib or with HD IL-2. One patient in each cohort experienced a grade 3 rash with vemurafenib. Typical HD IL2-related toxicities including constitutional symptoms and capillary leak syndrome were observed in both cohorts without excess cardiac, hepatic, pulmonary, renal or skin toxicities beyond that expected with this agent. No prolongation of QT interval was observed in patients treated with HD IL-2 in either cohort. There were no treatment related deaths.

Although our intent was to pursue predictive biomarker data, due to funding issues and early closure this endeavor was aborted. A shift in the melanoma treatment landscape during this trial adversely affected accrual, in that combination BRAF/MEK inhibitor therapy soon became an approved and common standard treatment approach, while durable responses were achieved in a substantial percentage of patients treated with checkpoint inhibitors, which led to early trial closure.

\section{Discussion}

Treatment options for advanced malignant melanoma have expanded significantly in the past number of years. When this trial was first conceptualized, in early 2011, those options were limited. HD IL-2 was the first systemic immunotherapy approved for patients with advanced melanoma but efficacy was observed in a select number of patients. Dramatic responses to the BRAF inhibitor, vemurafenib, and preclinical as well as early clinical reports of enhanced antigen expression and a favorable tumor microenvironment in patients treated with such targeted agents led to our attempt to combine this agent in BRAF-mutated melanoma [19-25]. Combination BRAF-MEK inhibitor therapy in this patient population was not yet approved when this trial

Table 3 Duration of Response (Days)

\begin{tabular}{llll}
\hline Response & & Cohort 1 & Cohort 2 \\
\hline Complete Response & Mean (SD) & $84(93.8)$ & - \\
& Median & 56 & - \\
& Range & $8-189$ & (ongoing) \\
Partial Response & Mean (SD) & $194(180.0)$ & $113(103.0)$ \\
& Median & 118 & 133 \\
Stable Disease & Range & $77-512$ & $1-204$ \\
& Mean (SD) & $125(156.8)$ & $127(186.3)$ \\
& Median & 56 & 63 \\
& Range & $1-509$ & $1-544$ \\
\hline
\end{tabular}




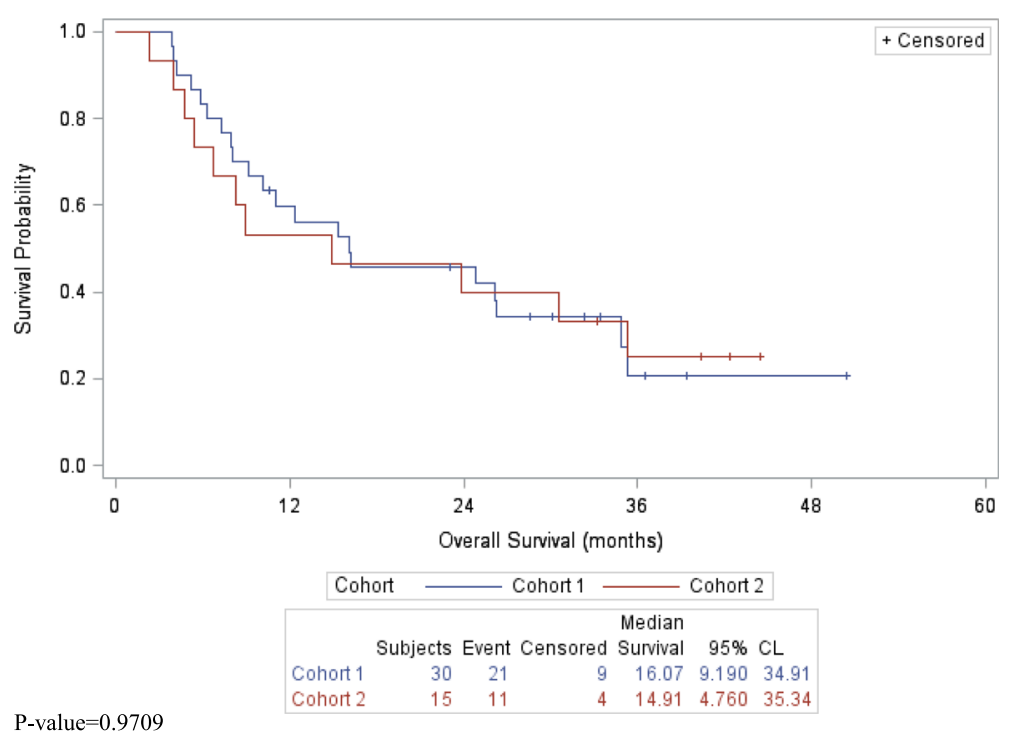

Fig. 3 Overall Survival by Cohort

initiated. Patient accrual to this study was negatively affected shortly after opening due to rapidly expanding treatment options for this group of patients, thus the trial closed to accrual prior to meeting its target population of 135 patients in Cohort 1 .

The safety results of this study show vemurafenib combined with HD IL-2 did not change the known safety profile of either drug. No dose reductions were required for vemurafenib and there were no treatment related deaths. The overall response rate of approximately $27 \%$ for each cohort observed at assessment 1 was lower than expected for unclear reasons. One would expect a response rate of $40-50 \%$ for vemurafenib alone [1, 2], whereas response rates to HD IL-2 alone are consistently roughly $15-28 \%$ [6, $28,29]$, although caution must be taken when comparing the current results to previously published data. Assessment 1 occurred at $10 \pm 3$ weeks from the start of HD IL-2. In Cohort 1, vemurafenib was taken for 6 weeks prior to starting HD IL-2, and in Cohort 2, vemurafenib was taken for 7 to 18 weeks prior to starting HD IL-2 but patients had to have had stable or responding disease before enrollment. Vemurafenib was held during each five-day cycle of inpatient HD IL-2 therapy in order to avoid excess toxicity especially hepatic or cardiac in nature. Systemic HD IL-2 may have adversely enhanced immunosuppression, e.g. via upregulating Treg function, which may help explain the

Table 4 1-, 2-, 3-year Survival

\begin{tabular}{lll}
\hline Survival & Cohort 1 $(N=30)$ & Cohort 2 $(N=15)$ \\
\hline 1 -year & $18(60 \%)$ & $8(53 \%)$ \\
2-year & $14(46.7 \%)$ & $6(40 \%)$ \\
3-year & $9(30 \%)$ & $4(26.7 \%)$ \\
\hline
\end{tabular}

dampening effect of vemurafenib therapy and may serve as one potential explanation for this lower than expected response rate. It is conceivable that if combination BRAF-MEK inhibitor therapy was used instead of single agent vemurafenib that observed response rates would have been higher. Biomarker assessment would enhance a better understanding of such an effect, but these assessments were not performed in this trial. Sullivan and colleagues have made an initial attempt at these evaluations in a similar smaller trial [30]. Disease control rate and 3-year overall survival, however, were as would be expected with HD IL-2 therapy alone in patients with advanced malignant melanoma [8, 27-29]. Thus this combination does not appear to induce and overall negative clinical outcome.

Combination targeted therapy with systemic immunotherapy is a valid approach worth investigation based on preclinical models and early clinical results [18-25, 31]. Caution must be heeded however as unexpected untoward toxicity may arise [32]. Active studies are ongoing using other BRAF/MEK combinations and immune checkpoint inhibitors, results of which are eagerly anticipated.

\section{Conclusions}

In conclusion, results of this trial reveal that the combination of vemurafenib and HD IL-2 in the treatment of BRAF-mutated metastatic malignant melanoma was safe but without added clinical benefit beyond what would be expected with either agent alone. Accrual to the study was halted far short of planned due to a dramatic shift in the treatment landscape of this previously poorly responsive disease. Future alternative combination approaches remain worthy investigative endeavors. 


\section{Acknowledgements}

The authors wish to thank the patients and their families who enrolled on this clinical trial, along with the clinical research staff at each participating institution. We would also thank Janice P. Dutcher, M.D. for editorial assistance in preparing this manuscript.

Presented in part at the 51st Annual meeting of the American Society of Clinical Oncology, May 29 - June 2, 2015, Chicago, IL.

\section{Funding}

The trial was sponsored by Prometheus Laboratories Inc., San Diego, CA.

\section{Availability of data and materials}

The datasets during and/or analyzed during study available from the corresponding author upon reasonable request.

\section{Authors' contributions}

JIC, TLL, SA, DHL made substantial contributions to conception and design, and acquisition of data. JIC, JS, SA - made substantial contributions to analysis and interpretation of data. JIC - wrote the original draft of the manuscript, all authors were involved in revising it critically for important intellectual content; gave final approval of the version to be published; agreed to be accountable for all aspects of the work in ensuring that questions related to the accuracy or integrity of any part of the work are appropriately investigated and resolved.

\section{Ethics approval and consent to participate}

Human investigations were performed after approval by an institutional review board or ethics committee at each participating institution and in accordance with an assurance filed with and approved by the U.S. Department of Health and Human Services. Written informed consent was obtained on all patients.

\section{Consent for publication}

Not applicable.

\section{Competing interests}

J.I. Clark: non-paid advisory role - PROCLIVITY registry, Prometheus; advisory board - Exelixis; speakers' bureau - Bristol-Myers Squibb, Merck; research funding to institution - Acceleron Pharma, Argos Therapeutics, AVEO, BristolMyers Squibb, Prometheus, Roche.

J. Singh: no competing interests reported.

M.S. Ernstoff: no competing interests reported.

C.D. Lao: research funding to institution: Bristol-Myers Squibb, Merck, GlaxoSmithKline, Norvatis; travel reimbursement: Bristol-Myers Squibb. L.E. Flaherty: non-paid DSMB role - Merck.

T.F. Logan: consulting/advisory role - Prometheus; research funding to institution - Abbott Laboratories, Abraxis BioScience, Acceleron Pharma, AMGEN, Argos Therapeutics, AstraZeneca, AVEO, BioVex, Bristol-Myers Squibb, Eisai, Lilly, GlaxoSmithKline, Roche, immatics, Merck, Novartis, Pfizer, Prometheus, Synta, Thrshold Pharmaceuticals, Millennium, TRACON Pharma, Cerulean, EMD Serono.

B. Curti: consulting/advisory role - Prometheus; research support -

Prometheus, Galectin Therapeutics, Viralytics; research funding to institution

- Bristol-Myers Squibb, Medlmmune; speakers' bureau - Prometheus, BristolMyers Squibb.

S.S. Agarwala: no competing interests reported.

B. Taback: no competing interests reported.

L. Cranmer: no competing interests reported.

J. Lutzky: no competing interests reported.

T.L. Luna: no competing interests reported.

S. Aung: no competing interests reported.

D.H. Lawson: no competing interests reported.

\section{Publisher's Note}

Springer Nature remains neutral with regard to jurisdictional claims in published maps and institutional affiliations.

\section{Author details}

'Cardinal Bernardin Cancer Center, Loyola University Medical Center, 2160 S. First Avenue, Maywood, IL 60153, USA. ²Primary Biostatistical Solutions, Victoria, BC, Canada. ${ }^{3}$ Roswell Park Cancer Institute, Buffalo, NY, USA.
${ }^{4}$ University of Michigan, Ann Arbor, MI, USA. ${ }^{5}$ The Karmanos Cancer Institute, Detroit, MI, USA. 'Indiana University, Indianapolis, IN, USA. 'Earle A. Chiles Research Institute, Providence Cancer Center, Portland, OR, USA. ${ }^{8}$ St. Luke's Hospital and Health Network, Bethlehem, PA, USA. ${ }^{9}$ Columbia University/ Herbert Irving Comprehensive Cancer Center, New York, NY, USA. ${ }^{10}$ Fred Hutchinson Cancer Research Center, University of Washington, Seattle, WA, USA. ${ }^{11}$ Mt. Sinai Comprehensive Cancer Center, Miami Beach, FL, USA.

${ }^{12}$ Prometheus Laboratories Inc, San Diego, CA, USA. ${ }^{13}$ Emory Winship Cancer Institute at Emory University, Atlanta, GA, USA. ${ }^{14}$ Nektar Inc, San Diego, CA, USA.

Received: 25 January 2018 Accepted: 6 July 2018

Published online: 27 July 2018

\section{References}

1. Chapman PB, Hauschild A, Robert C, Haanen JB, Ascierto P, Larkin J, et al. Improved survival with vemurafenib in melanoma with BRAF V600E mutation. N Engl J Med. 2011;364:2507-16.

2. Sosman JA, Kim KB, Schuchter L, Gonzalez R, Pavlick AC, Weber JS, et al. Survival in BRAF V600-mutant advanced melanoma treated with vemurafenib. N Engl J Med. 2012;366:707-14.

3. Hauschild A, Grob JJ, Demidov LV, Jouary T, Gutzmer R, Millward M, et al. Dabrafenib in BRAF-mutated metastatic melanoma: a multi-Centre, openlabel, phase 3 randomised controlled trial. Lancet. 2012;380:358-65.

4. Robert C, Karaszewska B, Schachter J, Rutkowski P, Mackiewicz A, Stroiakovski D, et al. Improved overall survival in melanoma with combined dabrafenib and tramentinib. N Engl J Med. 2015;372:30-9.

5. Larkin J, Ascierto PA, Dréno B, Atkinson V, Liszkay G, Maio M, et al. Combined vemurafenib and cobimetinib in BRAF-mutated melanoma. N Engl J Med. 2014;371:1867-76.

6. Atkins MB, Lotze MT, Dutcher JP, Fisher RI, Wess G, Margolin K, et al. Highdose recombinant interleukin 2 therapy for patients with metastatic melanoma: analysis of 270 patients treated between 1985 and 1993. J Clin Oncol. 1999;17:2105-16.

7. Atkins MB, Kunkel L, Sznol M, Rosenberg SA. High-dose recombinant interleukin-2 therapy in patients with metastatic melanoma: long-term survival update. Cancer J Sci Am. 2000;6(Suppl 1):S11-4.

8. Alva A, Daniels GA, Wong MKK, Kaufman HL, Morse MA, McDermott DF, Clark Jl, Agarwala SS, Miletello G, Logan TF, Hauke RJ, Curti B, Kirkwood JM, Gonzalez R, Amin A, Fishman M, Agarwal N, Lowder JN, Hua H, Aung S, Dutcher JP. Contemporary experience with high-dose interleukin-2 therapy and impact on survival in patients with metastatic melanoma and metastatic renal cell carcinoma. Cancer Immuno Immunother. 2016;65:1533-44.

9. Hodi FS, O'Day SJ, McDermott DF, Weber RW, Sosman JA, Haanen JB, et al. Improved survival with ipilimumab in patients with metastatic melanoma. N Engl J Med. 2010;363:711-23.

10. Schadendorf D, Hodi FS, Robert C, Weber JS, Margolin K, Hamid O, et al. Pooled analysis of long-term survival data from phase II and phase III trials of ipilimumab in unresectable or metastatic melanoma. J Clin Oncol. 2015; 33:1889-94.

11. Topalian SL, Hodi FS, Brahmer JR, Gettinger SN, Smith DC, McDermott DF, et al. Safety, activity, and immune correlates of anti-PD-1 antibody in cancer. N Engl J Med. 2012;366:2443-54

12. Hamid O, Robert C, Daud A, Hodi FS, Hwu WJ, Kefford R, et al. Safety and tumor responses with lambrolizumab (anti-PD-1) in melanoma. N Engl J Med. 2013;369:134-44

13. Robert C, Schachter J, Long GV, Arance A, Grob JJ, Mortier L, et al. Pembrolizumab versus ipilimumab in advanced melanoma. N Engl J Med. 2015:372:2521-32.

14. Wolchok JD, Kluger H, Callahan MK, Postow MA, Rizvi NA, Lesokhin AM, et al. Nivolumab plus ipilimumab in advanced melanoma. N Engl J Med. 2013; 369:122-33.

15. Postow MA, Chesney J, Pavlick AC, Robert C, Grossman K, McDermott D, et al. Nivolumab and ipilimumab versus ipilimumab in untreated melanoma. $\mathrm{N}$ Engl J Med. 2015;372:2006-17.

16. Larkin J, Chiarion-Sileni V, Gonzalez R, Grob JJ, Cowey CL, Lao CD, Schadendorf $\mathrm{D}$, et al. Combined nivolumab and ipilimumab or monotherapy in untreated melanoma. N Engl J Med. 2015;373:23-34.

17. Gide TN, Womott JS, Scolyer RA, Long GV. Primary and acquired resistance to immune checkpoint inhibitors in metastatic melanoma. Clin Cancer Res. 2018;24:1260-70. 
18. Sumimoto H, Imabayashi F, Iwata T, Kawakami Y. The BRAF-MAPK signaling pathway is essential for cancer-immue evasion in human melanoma cells. J Exp Med. 2006;203:1651-6.

19. Kono M, Dunn IS, Durda PJ, Butera D, Rose LB, Haggerty TJ, et al. Role of mitogen-activated protein kinase signaling pathway in the regulation of human melanocytic antigen expression. Mol Cancer Res. 2006:4:779-92.

20. Frederick DT, Piris A, Cogdill AP, Cooper ZA, Lezcano C, Ferrone CR, et al. BRAF inhibition is associated with enhanced melanoma antigen expression and a more favorable tumor microenvironment in patients with metastatic melanoma. Clin Cancer Res. 2013;19:1225-31.

21. Boni A, Cogdill AP, Dang P, Udayakumar D, Njauw CN, Sloss CM, et al. Selective BRAF ${ }^{\mathrm{V} 600 \mathrm{E}}$ inhibition enhances T-cell recognition of melanoma without affecting lymphocyte function. Cancer Res. 2010;70:5213-9.

22. Koya RC, Mok S, Otte N, Blacketor KJ, Comin-Anduix B, Tumeh PC, et al. BRAF inhibitor vemurafenib improves the antitumor activity of adoptive cell immunotherapy. Cancer Res. 2012;72:3928-37.

23. Liu C, Peng W, Xu C, Lou Y, Zhang M, Wargo JA, et al. BRAF inhibition increases tumor infiltration by $T$ cells and enhances the antitumor activity of adoptive immunotherapy in mice. Clin Cancer Res. 2013;19:393-403.

24. Comin-Anduix B, Chodon T, Sazegar H, Matsunaga D, Mock S, Jalil J, et al. The oncogenic BRAF kinase inhibitor PLX4032/RG7204 does not affect the viability or function of human lymphocytes across a wide range of concentrations. Clin Cancer Res. 2010;16:6040-8.

25. Whipple CA, Boni A, Fisher JL, Hampton TH, Tsongalis GJ, Mellinger DL, Yan S, Tafe L, Brinckerhoff CE, Turk MJ, Mullins DW, Fadul CE, Ernstoff MS. The mitogen-activated protein kinase pathway plays a critical role in regulating immunological properties of BRAF mutant cutaneous melanoma cells. Melanoma Res. 2016;26:223-35.

26. Wolchok JD, Hoos A, O'Day S, Weber JS, Hamid O, Lebbé C, et al. Guidelines for the evaluation of immune therapy activity in solid tumors: immunerelated response criteria. Clin Cancer Res. 2009;15:7412-20.

27. Sullivan RJ, Hoshida Y, Logan T, Khushalani NI, Giobbie-Hurder A, Margolin K, Roder J, Bhatt R, Koon H, Olencki T, Hutson T, Curti B, Blackmon S, Mier J, Roder H, Stewart J, Amin A, Ernstoff M, Clark J, Atkins MB, Kaufman H, Sosman J, Signoretti S, McDermott DF. High dose interleukin 2 (HD IL-2) select trial in melanoma: a tissue and blood collection protocol to identify predictive biomarkers of response to HD IL-2 in patients with advanced melanoma. J Immunother Cancer. 2016;4(suppl 2):P6.

28. Wong MKK, Kaufman HL, Daniels GA, McDermott DF, Aung S, Lowder JN, Morse MA. Implementation of an interleukin-2 national registry: an opportunity to improve cancer outcomes. J Immunother Cancer. 2014;2:20-2.

29. Payne R, Glenn L, Hoen H, Richards B, Smith JW 2nd, Lufkin R, et al. Durable responses and reversible toxicity of high-dose interleukin-2 treatment of melanoma and renal cancer in a community hospital biotherapy program. J Immunother Cancer. 2014;2:2-13.

30. Mooradian MJ, Reuben A, Prieto PA, Hazar-Rethinam M, Frederick DT, Nadres B, Piris A, Juneja V, Cooper ZA, Sharpe AH, Corcoran RB, Flaherty KT, Lawrence DP, Wargo JA, Sullivan RJ. A phase II study of combined therapy with a BRAF inhibitor (vemurafenib) and interleukin-2 (aldesleukin) in patients with metastatic melanoma. Oncoimmunol. 2018:7(5):e1423172.

31. Deniger DC, Kwong MLM, Pasetto A, Dudley ME, Wunderlich JR, Langhan MM, et al. A pilot trial of the combination of vemurafenib with adoptive cell therapy in patients with metastatic melanoma. Clin Cancer Res. 2016;23:351-62.

32. Ribas A, Hodi FS, Callahan M, Konto C, Wolchok J. Hepatotoxicity with combination of vemurafenib and ipilimumab. N Engl J Med. 2013;368:1365-6.

\section{Ready to submit your research? Choose BMC and benefit from:}

- fast, convenient online submission

- thorough peer review by experienced researchers in your field

- rapid publication on acceptance

- support for research data, including large and complex data types

- gold Open Access which fosters wider collaboration and increased citations

- maximum visibility for your research: over $100 \mathrm{M}$ website views per year

At BMC, research is always in progress.

Learn more biomedcentral.com/submissions 